\title{
Transcriptomic profiling of canine atrial fibrillation models after one week
}

\section{of sustained arrhythmia}

Francis J.A. Leblanc, BS ${ }^{1,2}$, Faezeh Vahdati Hassani, $\mathrm{MS}^{1,2}$, Laura Liesinger, $\mathrm{MS}^{3,4}$, Xiaoyan

Qi, $P h D^{2}$, Patrice Naud, $\mathrm{PhD}^{2}$, Ruth Birner-Gruenberger, $\mathrm{PhD}^{3,4,5}$, Guillaume Lettre, $\mathrm{PhD}^{1,2 \star}$, Stanley Nattel, $\mathrm{MD}^{1,2,6 *}$

*Share senior authorship.

Running title: Transcriptomic analysis of AF canine models

\section{Affiliations}

${ }^{1}$ Faculty of Medicine, Université de Montréal, Montreal, Quebec, Canada

${ }^{2}$ Montreal Heart Institute, Montreal, Quebec, Canada

${ }^{3}$ Medical University of Graz, Diagnostic and Research Institute of Pathology, Graz, Austria ${ }^{4}$ BioTechMed-Graz, Omics Center Graz, Austria

${ }^{5}$ Technische Universität Wien, Institute of Chemical Technologies and Analytical Chemistry, Vienna, Austria

${ }^{6}$ Institute of Pharmacology, West German Heart and Vascular Center, Faculty of Medicine, University Duisburg-Essen, Essen, Germany

Correspondence to: Guillaume Lettre, PhD or Stanley Nattel, MD; 5000 Belanger St. E., Montreal, Quebec, Canada H1 T1C8. guillaume.lettre@umontreal.ca or Stanley.Nattel@icm-mhi.org Journal Subject Terms: Arrhythmias, Atrial Fibrillation, Electrophysiology, Catheter Ablation 


\section{ABSTRACT (250 words)}

Background: Atrial fibrillation (AF), the most common sustained arrhythmia, is associated with increased morbidity, mortality, and health-care costs. AF develops over many years and is often related to substantial atrial structural and electrophysiological remodeling. AF may lack symptoms at onset and atrial biopsy samples are generally obtained in subjects with advanced disease, so it is difficult to study earlier-stage pathophysiology in humans.

Methods: Here, we characterized comprehensively the transcriptomic (miRNAseq and mRNAseq) changes in the left atria of two robust canine AF-models after one week of electrically-maintained AF, without or with ventricular rate-control via atrioventricular nodeablation/ventricular pacing.

Results: Our RNA-sequencing experiments identified thousands of genes that are differentially expressed, including a majority that have never before been implicated in AF. Gene-set enrichment analyses highlighted known (e.g. extracellular matrix structure organization) but also many novel pathways (e.g. muscle structure development, striated muscle cell differentiation) that may play a role in tissue remodeling and/or cellular transdifferentiation. Of interest, we found dysregulation of a cluster of non-coding RNAs, including many microRNAs but also the MEG3 long non-coding RNA orthologue, located in the syntenic region of the imprinted human DLK1-DIO3 locus. Interestingly (in the light of other recent observations), our analysis identified gene-targets of differentially expressed microRNAs at the DLK1-DIO3 locus implicating glutamate signaling in AF pathophysiology.

Conclusions: Our results capture molecular events that occur at an early stage of disease development using well-characterized animal models, and may therefore inform future studies that aim to further dissect the causes of $A F$ in humans. 
Keywords: Atrial fibrillation, Atrial remodeling, Canine models, Transcriptomics, miRNA targets, Glutamate signaling, DLK1-DIO3 locus, MEG3.

\section{Nonstandard abbreviations and acronyms}

\section{CTL Control}

$\mathrm{AF}+\mathrm{AVB} \quad$ Atrial tachypacing with atrioventricular-node ablation

AF Atrial tachypacing without atrioventricular-node ablation

RAA Right atrial appendage

LA Left atrium

DE Differential expression

GSEA Gene set enrichment analysis

IncRNA Long non-coding RNA

\section{INTRODUCTION}

Atrial fibrillation $(\mathrm{AF})$ is the most common sustained arrhythmia, with an estimated lifetime risk of $22 \%-26 \%$ and association with increased morbidity and mortality ${ }^{1}$. Despite advances in antiarrhythmic therapies, their suboptimal efficacy and adverse effects have limited their use ${ }^{2}$. Therefore, there is a need to further characterize fundamental arrhythmia mechanisms in order to discover new therapeutic targets ${ }^{2}$. Although AF is known to be a final common endpoint of atrial remodeling resulting from a variety of heart diseases, it can also be, in turn, a cause of remodeling. This vicious cycle is called "AF begets $\mathrm{AF}^{33}$ and explains the progressive nature of this arrhythmia and the complexity of its management.

Atrial remodeling is characterized by ion channel dysfunction, $\mathrm{Ca}^{2+}$ handling abnormalities, and structural changes, which result in $\mathrm{AF}$ induction and maintenance ${ }^{4,5}$. Heart disease, and 
even rapid atrial activity itself, cause the development of atrial fibrosis, which is a hallmark of structural remodeling. The degree of fibrosis is positively correlated with the persistence of $\mathrm{AF}^{6}$. Atrial cardiomyocytes subjected to rapid activation release factors that induce fibroblastto-myofibroblast differentiation that leads to increased collagen synthesis ${ }^{7}$.

Any arrhythmia causing a rapid ventricular rate, including AF, is a well-recognized inducer of ventricular dysfunction, so-called "arrhythmia-induced cardiomyopathy". . Heart failure enhances atrial stretch and sympathetic tone, making AF more resistant to rate- or rhythmcontrol treatments ${ }^{9}$. AF promotion results from the rapid atrial rate, but rapid ventricular rates due to inadequate rate-control also promote AF-related atrial remodeling with a different profile from the remodeling produced by rapid atrial rate alone ${ }^{10}$. Radiofrequency atrioventricular node ablation (AVB) with right ventricular pacing is a nonpharmacological strategy for rate control that can improve symptoms and outcomes ${ }^{11}$.

Our previous work in canine AF models showed that maintaining AF for one week by rapid atrial pacing activates fibroblasts, collagen gene expression and cardiomyocyte ion channel changes, without yet causing fibrosis ${ }^{12}$. Continued electrical maintenance of AF for 3 weeks produces fibrosis, but electrically-maintained AF with ventricular rate control through AVB produces less profibrillatory remodeling than 3 weeks of $A F$ alone ${ }^{10}$. However, how AF with and without AVB impact atrial remodeling at the molecular level has not yet been assessed comprehensively. To answer this question, we took advantage of our well-characterized AF dog models and performed RNA-sequencing (RNA-seq) of cardiomyocyte-enriched atrial samples after one week to capture the molecular actors of atrial remodeling. In comparison with control (CTL) dogs, we found thousands of mRNAs, long non-coding RNA (IncRNA) and 
bioRxiv preprint doi: https://doi.org/10.1101/2021.02.04.429512; this version posted October 19,2021. The copyright holder for this preprint (which was not certified by peer review) is the author/funder, who has granted bioRxiv a license to display the preprint in perpetuity. It is made available under aCC-BY-NC-ND 4.0 International license.

microRNA (miRNA) that are differentially expressed (DE) in the atria of the canine AF models. Pathway analyses of the transcriptomic data highlighted known biological processes, but also potential novel modulators of arrhythmia initiation which may shed new light on our understanding of $\mathrm{AF}$ in humans. 


\section{METHODS}

All results and $\mathrm{R}$ code are available at https://github.com/lebf3/DogAF.

\section{Canine atrial fibrillation model}

A total of 18 adult mongrel dogs of either sex, weighing $18-32 \mathrm{~kg}$, were obtained from LAKA Inc and randomly assigned to control (CTL) group $(n=6)$ and two canine AF-models ( $n=6 /$ group) (Supplemental Table I). We selected 6 animals per group based on previously published RNAseq studies and our previous experiments with these models. Further, the number of DE genes identified in our analyses (post hoc) indicates that this sample size is sufficient to capture the main transcriptional changes that occur in the atrium of these dog models. Animals were handled in accordance with the "Guide for the Care and Use of Laboratory Animals" established by the National Institutes of Health as approved by the Montreal Heart Institute Ethics Committee (2016-47-01, 2019-47-03 for control dogs, 2015,47-01, 2018.47.12 for AF dogs).

To induce $A F$, animals were subjected to atrial tachypacing without $(\mathrm{AF})^{13}$ and with $(\mathrm{AF}+\mathrm{AVB})^{14}$ atrioventricular-node ablation under $0.07 \mathrm{mg} / \mathrm{kg}$ acepromazine $(\mathrm{IM}), 5.3 \mathrm{mg} / \mathrm{kg}$ ketamine (IV), and $0.25 \mathrm{mg} / \mathrm{kg}$ diazepam (IV), and $1.5 \%$ isoflurane anesthesia. In the AF group, a bipolar pacing lead with fluoroscopic guidance was placed in the right atrial appendage (RAA). In the AF+AVB group, pacing leads were inserted into the RAA and right ventricular apex. Pacing leads were connected to a subcutaneous pacemaker implanted in the neck (right side). In the AF+AVB group, radiofrequency catheter ablation was used to create $A F+A V B$. For this purpose, a quadripolar catheter with fluoroscopic guidance was placed across the tricuspid valve via the right femoral vein. Radiofrequency energy was then used to perform ablation when action potential at the His bundle was detected. Twenty-four to 
seventy-two hours after surgery, dogs in the AF group were subjected to AF-maintaining atrial tachypacing at 600 bpm for seven days. In the AF+AVB group, RA and right ventricle were paced at 600 and 80 bpm, respectively. In animals of the CTL group, no pacemaker was inserted. No adverse event was recorded and no dog was excluded.

\section{Enrichment of dog atrial cardiomyocytes}

Cardiomyocytes were enriched from the left atrium (LA) with enzymatic digestion through the coronary artery-perfused Langendorff system, as previously described ${ }^{15}$. Briefly, dogs were anesthetized with $2 \mathrm{mg} / \mathrm{kg}$ morphine (IV) and $120 \mathrm{mg} / \mathrm{kg}$ alpha-chloralose and mechanically ventilated. Hearts were aseptically and quickly removed after intra-atrial injection of $10,000 \mathrm{U}$ heparin and placed in Tyrode's solution containing $136 \mathrm{mM} \mathrm{NaCl}, 5.4 \mathrm{mM} \mathrm{KCl,} 2 \mathrm{mM} \mathrm{CaCl}$, $1 \mathrm{mM} \mathrm{MgCl}$, $10 \mathrm{mM}$ dextrose, $5 \mathrm{mM}$ HEPES, $0.33 \mathrm{NaH} 2 \mathrm{PO} 4$ (pH was adjusted to 7.3 with $\mathrm{NaOH}$ ). The left coronary artery of the isolated heart was cannulated, and the LA was dissected free and perfused with $100 \%$ oxygenated Tyrode's solution $\left(37^{\circ} \mathrm{C}, 1.8 \mathrm{mM} \mathrm{Ca}^{2+}\right)$. The arterial branches were ligated to have a leak-free system, and LA tissues were perfused with $\mathrm{Ca}^{2+}$-free Tyrode's solution for $\sim 10$ minutes, followed by $\sim 1$-hour perfusion with $\sim 0.45$ $\mathrm{mg} / \mathrm{mL}$ collagenase (CLSII, Worthington, Lakewood, $\mathrm{NJ}$ ) and $0.1 \%$ bovine serum albumin (Sigma-Aldrich, Oakville, ON) in $\mathrm{Ca}^{2+}$-free Tyrode's solution for enzyme digestion. Digested tissue was removed from the cannula and cut into small pieces, and atrial cardiomyocytes were harvested. 


\section{RNA-seq/miRNA-seq}

\section{Library preparation and sequencing}

mRNA and miRNA libraries were prepared at Genome Québec. mRNA libraries were made with the NEBNext_dual kit (rRNA-depleted stranded) and sequenced on NovaSeq 6000 S2 PE100 Illumina platform generating 32-123M Paired-end reads per sample. miRNA libraries were prepared with TruSeq smRNA and sequenced on the HiSeq 4000 SR50 Illumina platform generating $10-12 \mathrm{M}$ reads per sample.

\section{Bioinformatic processing and DE analysis}

The complete analysis can be found at https://github.com/lebf3/Dog_AF_transcriptomic. Briefly, mRNA reads were pseudomapped on reference transcriptome CanFam3.1.98 with Kallisto ${ }^{16}$ with the options quant -t 5 -b 100 and the rest as default. We aggregated transcripts by genes with tximport ${ }^{17}$ and quantified with DESeq $2^{18}$. Genes with 0 reads in more than 12 samples were removed. Shrunken $\log _{2}$ transformed expression corrected for library size, with and without fibroblast fraction as a covariate (within DEseq2's model) were then analyzed for DE with Wald test for all pairwise comparisons of $C T L, A F$ and $A F+A V B$ and likelihood ratio test for total assay DE. We did not adjust our differential gene expression analyses for biological sex because no genes where DE between female and male dogs in our experiment. We plotted the PCA with fibroblast fraction as a covariate from $\log _{2}$ transformed expression values corrected with Limma's removeBatchEffect() function ${ }^{19}$ for visualization of fibroblast effect on the top 1000 most variable genes. We then compared sets of GENEIDs found to be up (L2FC $>0 \& p<0.01)$ or down ( $22 \mathrm{FC}<0 \& p<0.01)$ in all possible contrasts. 
For miRNAs, we trimmed reads using fastp ${ }^{20}$ with default settings and aligned them to CanFam3.1.98 genome with STAR v2.7.1 $\mathrm{a}^{21}$ according to ENCODE protocol ${ }^{22}$. DEseq2 DE analysis was then conducted with the same parameters as described above for mRNAs.

\section{Deconvolution of RNA-seq data}

To account for potential tissue heterogeneity, we used a murine atrial gene signature matrix described in Donovan et al. ${ }^{23}$ and our matrix of gene expression in Fragments Per Kilobase of exon model per Million reads mapped (FPKM) in CIBERSORTx online tool ${ }^{24}$. We then performed nonparametric Wilcoxon test on all possible comparisons for fibroblast fraction with a statistical significance threshold of $p<0.05$.

Gene set enrichment analyses

For each gene sets described above, we performed hypergeometric testing against the human Gene Ontology (GO) Biological Processes (BP) from Molecular Signatures Database v7.1 with the HypeR package.

miRNA target prediction

For DE miRNA present in the 5 most cited miRNA databases (DIANA, Miranda, PicTar, TargetScan, and miRDB), we defined genes as targets if they were: i-annotated with a human homolog in the ensemble database, ii-predicted targets by at least 3 out of 5 databases queried with the MiRNAtap package, iii-DE (mRNA FDR < 0.01), iv-inversely correlated (Pearson's $r<-0.5) \log _{2}$ expression, corrected for the fibroblast fraction (expression values corrected with Limma's removeBatchEffect() function). We then performed a GSEA with the 
remaining 82 predicted targets of the miRNA located on the syntenic region of the DIk1-Dio3 locus (CanFam3.1 Chr8:68961744-69696779) as described above.

RNA-seq and miRNA-seq DE genes comparison between human AF patients and canine AF models

DE genes in our canine AF models with annotated human orthologues in the ENSEMBL database were compared to a meta-analysis of miRNA DE in human AF and a large RNAseq study on left atrial appendages obtained from 261 patients undergoing valve surgery ${ }^{25,26}$. Precursors of the human miRNAs listed in Shen et al. Table S8 ( $n=53,21$ upregulated and 32 downregulated) ${ }^{25}$ were retrieved with the $\mathrm{R}$ package miRBaseConverter and then compared across species. Because only 5 miRNA were found to overlap with human DE miRNA, only mRNA genes found to be DE in human and our canine AF models are represented as an Upset plot. The counts for human mRNA data were downloaded from GEO database (GSE69890). DE testing was conducted as described above for the 3 groups; no AF (CTL, $n=50), A F$ in AF rhythm (AF, $n=130$ ), and $A F$ in sinus rhythm (AF.SR, $n=81$ ), with inclusion of sex as a covariate.

\section{Mitochondrial genes DE in canine AF models}

The human MitoCarta3. $0^{27}$ database was queried for genes with mitochondrial localization in the heart $(n=539)$. We represented DE genes with likelihood ratio test FDR $<0.01$ from that list as volcano plots. 


\section{Proteomics}

Dog cardiomyocytes were lysed by sonication, reduced and alkylated. Protein was precipitated, resuspended, quantified and subjected to tryptic digest. Peptides (500 ng) were analyzed by reverse phase nano-HPLC coupled to a Bruker maXis II mass spectrometer (positive mode, mass range $150-2200 \mathrm{~m} / \mathrm{z}$, collision induced dissociation of top 20 precursors). LC-MS/MS data were analyzed for protein identification and label-free quantification using MaxQuant ${ }^{28}$ (1.6.1.0) against the public database UniProt with taxonomy Canis lupus familiaris and common contaminants (downloaded on 01.08.2019, 29809 sequences) with carbamidomethylation on Cys as fixed and oxidation on Met as variable modification with decoy database search included (mass tolerance 0.006 Da for precursor, 80 ppm for product ions; $1 \%$ PSM and protein FDR, match between runs enabled, minimum of 2 ratio counts of quantified razor and unique peptides).

\section{DE analysis and correlation}

Proteins with $>3$ missing values per treatment were removed. The remaining missing intensities were replaced with random values taken from the Gaussian distribution centered around a minimal value from the 10th quantile with the DEP package's Minprob function, to simulate a relative label-free quantification (LFQ) value for those low abundant proteins. Twosample t-tests with subsequent multiple testing correction by FDR were used to identify DE proteins $(p<0.01)$ with the fibroblast fraction as covariate using the Limma package.

Because proteomic processing does not always converge to a single protein, only 755 genes out of the 1029 in the proteomic matrix were correlated to their corresponding RNA-seq data. We compared overlapping genes' mean $\log _{2}$ transformed expression in proteomic and RNA- 
bioRxiv preprint doi: https://doi.org/10.1101/2021.02.04.429512; this version posted October 19,2021 . The copyright holder for this preprint (which was not certified by peer review) is the author/funder, who has granted bioRxiv a license to display the preprint in perpetuity. It is made available under aCC-BY-NC-ND 4.0 International license.

seq. The distribution of mean RNA-seq expression of the 755 overlapping genes was then compared to the full mean RNA-seq gene expression values. 


\section{RESULTS}

\section{RNA-sequencing of cardiomyocyte-enriched atrial samples from canine AF models}

We analyzed data from three groups of six dogs. The first group (CTL) was the control group without atrioventricular ablation (AVB) nor pacemaker, in the second group (AF), right atrialtachypacing at 600 beats per minute (b.p.m.) was used to maintain AF electrically for one week, and the third group (AF+AVB) included dogs with electrically-maintained $A F$ for one week in the presence of $A V B$ and ventricular pacing at 80 b.p.m. to control the ventricular rate. We reasoned that transcriptomic profiling of atria from these animals should allow us to discover the molecular changes that occur over the first week after the onset of AF, and play a role in the development of the tissue remodeling accompanying the transition from paroxysmal to persistent AF.

Initial analysis of bulk RNA-seq data hinted at some heterogeneity of cellular composition across samples. Therefore, we estimated the fraction of the major cell types in each sample using an in-silico deconvolution technique implemented in CIBERSORTx (Fig. 1A) ${ }^{24}$. Because of the induced tissue remodeling due to the AF treatments, we found that both AF and $A F+A V B$ dogs had more fibroblasts in their atria than CTL animals (Fig. 1B). To emphasize the transcriptional differences between conditions that are not a result of variable cellular composition, we included the fibroblast fraction as a covariate in all subsequent DE analyses. Correction for this confounding variable reduced inter-group variability (Fig. 1C-D). 


\section{Proteomic analysis largely confirms the transcriptomic results}

To validate our RNA-seq results, we took advantage of mass spectrometry (MS)-based protein quantification results from the same 18 dog atrial cardiomyocyte-enriched cell extractions that were generated in a parallel study (detailed proteomic results will be presented elsewhere). After stringent quality control, we obtained relative quantification for 755 proteins. For these genes, the relative RNA and protein levels were strongly correlated (Pearson's $r=0.49, P=1.57 \times 10^{-46}$ ) (Fig. 2A). Many of the genes that are well-correlated encode abundant cardiomyocyte proteins, such as titin (TTM), myosin light chain-4 (MYL4), desmin (DES), and tropomyosin-1 (TPM1). We found that RNA-seq could profile transcripts with a wider range of expression profiles, whereas MS-based proteomics preferentially captured proteins whose genes are expressed at high levels. (Fig. 2B).

\section{Transcriptomic changes in cardiomyocyte-enriched atrial samples}

Pairwise comparisons of gene expression levels between the three groups of dogs identified 434, 5971, and 7867 genes that are DE (false discovery rate $(F D R)<0.01)$ in atrial cardiomyocyte-rich fractions in AFvsCTL, AF+AVBvsCTL, and AFvsAF+AVB, respectively (Fig. 3A-B). All differential gene expression level results are available in Supplemental Table II and https://github.com/lebf3/DogAF). Many genes previously implicated in AF are dysregulated in both $A F$ and $A F+A V B$ dogs when compared to controls, thus validating the experimental design. This includes $F H L 1$ involved in myofilament regulation ${ }^{29}$, SORBS2 involved in intercalated disc gap junction regulation ${ }^{30}$, and $K C N A 5$, which regulates atrial action potential repolarization ${ }^{31}$. Previous studies have established an important role for mitochondrial dysfunction in the etiology of $\mathrm{AF}^{27}$. Accordingly, we identified 54 genes that encode mitochondrial proteins that are DE in our AF canine models (Supplemental Figure I). 
In particular in the AF+AVB group, we noted the up-regulation of two key beta-oxidation genes (CPT1A and $A C A D L)$ and the down-regulation of the electron transport chain genes COX17 and NDUFA8. However, our data also implicates genes not previously recognized to be involved in AF, such as leukocyte receptor cluster member-8 (LENG8), transcription elongation regulator-1 (TCERG1), ligand dependent nuclear receptor corepressor $(L C O R)$, formin-binding protein-4 (FNBP4), and ENSCAFG00000049959 (orthologue of the IncRNA MEG3)(Fig. 3A, Supplemental Table III, and https://github.com/lebf3/DogAF).

To understand what pathways are modulated in the atria of these canine AF models, we performed gene set enrichment analyses (GSEAs) on the DE genes (Fig. 3C and Supplemental Table IV). In AFvsCTL, we noted an up-regulation of genes associated with profibrotic pathways (e.g. extracellular structure organization, biological adhesion, response to wounding) and a down-regulation of genes implicated in angiogenesis, such as blood vessel morphogenesis. Genes implicated in muscle biology were up-regulated in the AF+AVBvsCTL analysis (e.g. muscle structure development, striated muscle cell differentiation) whereas the same comparison implicated down-regulated genes involved in ion transport and signaling pathways (e.g. sensory perception). We confirmed that this enrichment was not due to a smaller fraction of cardiac neurons found in the atria of $A F+A V B$ dogs (Kruskal-Wallis' $P=0.32$ ). Because of the large overlap in genes that are down-regulated in $A F+A V B v s C T L$ and up-regulated in AFvsAF+AVB (Fig. 3B), we identified similar pathways in the GSEA for these two comparisons (in Fig. 3C, compare AF+AVBvsCTL and $A F v s A F+A V B)$. Finally, genes that were down-regulated in the AFvsAF+AVB analysis implicated genes with more generic functions in gene expression and chromatin modifications, such as the histone-lysine N-methyltransferase SETD5 and the DNA 
methyltransferase TET2. Dysregulation of the expression of these chromatin-related genes and pathways is consistent with the extensive transcriptomic changes observed in the atria of AF+AVB dogs, in sheep models of AF (Supplemental Figure II) as well as in AF patients ${ }^{32,}$ 33

\section{Dysregulation of miRNA expression}

Because miRNA play important roles in AF biology ${ }^{34}$ but are not detected in standard RNAseq protocols, we performed in parallel miRNA-seq on the same dog samples. We found 31, 19 and 21 miRNA that are DE (FDR <0.01) in AFvsCTL, AF+AVBvsCTL and AFvsAF+AVB, respectively (Fig. 4A, Supplemental Table II). When comparing miRNA expression in the two AF models, MIR185 on the dog chromosome 26 was the most DE miRNA with strong upregulation in the atria of AF animals. We also noted that 11 of the most strongly DE miRNA in the AF+AVBvsCTL and AFvsCTL analyses (MIR136, MIR411, MIR370, MIR127, MIR493, MIR494, MIR485, ENSCAFG00000025655 (96.20\% identity to hsa-mir-379), MIR758, MIR543, MIR889) mapped to the chr8:68,900,000-69,700,000 region in the dog reference genome CanFam3.1 (Fig. 4B). This region, highly conserved in mammals, is syntenic to the imprinted $14 \mathrm{q} 32$ region in humans (also known as the DLK1-DIO3 locus) ${ }^{35}$. The IncRNA MEG3, which we described above as being over-expressed in the AF canine models is also located in the same DLK1-DIO3 syntenic dog locus.

The dysregulation of the expression of IncRNA and miRNA at the same locus suggested that they might co-regulate the expression of genes implicated in the same biological pathway(s). To address this possibility, we used in silico predictions to infer the DE mRNA that are possible direct targets of these DE miRNA located at the syntenic DLK1-DIO3 locus. For this 
analysis, we focused on DE miRNAs and DE mRNAs that are predicted to physically interact by at least three out of five databases and that have expression levels that are negatively correlated in the RNA-seq/miRNA-seq experiments (Pearson's $r<-0.5$ ). Using these filters, we identified 82 potential target genes for the DE miRNAs at this locus, with most genes targeted by a single miRNA (Fig. 4C). GSEA with these 82 genes indicated a common role in synaptic signaling involving glutamate signaling (Fig. 4D and Supplemental Table V). Some of the key genes within these pathways are metabotropic glutamate receptor-1 and -8 (GRM1, GRM8), glutamate ionotropic receptor delta type subunit-1 (GRID1), glutamate ionotropic receptor AMPA type subunit-1 (GRIA1), and corticotropin-releasing factor-binding protein $(C R H B P)$.

\section{Partial differential transcriptomic overlap between human and dog AF atrial samples}

To assess the ability of our canine AF models to capture early transcriptomic changes which might be missed by profiling the atria of human AF patients who have developed the disease over years, we compared the DE genes identified in AF dogs with results from human left atrial transcriptomic profiling experiments ${ }^{25,26}$. Hsu et al. performed bulk RNA-seq experiments on left atrial appendages from 261 patients who underwent cardiac surgery to treat AF, valve disease, or other cardiac disorders. For differential gene expression analyses, these patients were divided between no $A F(n=50), A F$ in $A F$ rhythm $(n=130)$, and AF in sinus rhythm $(n=81)$. When we intersected this list of human DE genes with the list of DE genes in our AF dog models (with clear human orthologs), we identified 668 genes (Fig. 5 and Supplemental Table VI). We found the strongest overlap between dog AF+AVB and human AF in AF rhythm. Of note, most of the strongest signals in our dog study are also present in this human study (LENG8, SORBS2, BMP10, FNBP4 and glutamate receptor-related genes 
(GRM1, GRM8, GRIA1, GRIK2, GRID1)). For miRNA, we compared our results with data from a large meta-analysis involving 40 articles and 283 DE miRNA in AF (in different tissues and species) ${ }^{25}$. Of the 53 AF-associated miRNAs that were previously identified in human cardiac tissues and showed consistent results in the meta-analysis, we found five miRNAs in our analyses of the dog transcriptomic datasets (MIR144, MIR142, MIR146B, MIR223 and MIR451). These miRNAs have not been characterized functionally yet for a role in AF. Generally, the canine AF group matched better the directionality of change reported in the human AF DE miRNA meta-analysis. 


\section{DISCUSSION}

In this study, we used a transcriptomic approach to comprehensively assess the molecular architecture of AF-induced remodeling with and without AVB from atria cardiomyocyteenriched samples. We validated the robustness of our RNAseq data by correlating it with a proteomic analysis, which showed a strong correlation in gene expression, with well-defined cardiomyocyte genes being most highly expressed in both datasets (e.g. TTN, MYL4). We confirmed the involvement of known AF factors like the reactivation of developmental pathways, but also found a strong and novel association with microRNAs and IncRNA from the DLK1-DIO3 locus, including the MEG3 canine orthologue. This finding is concordant with the many chromatin remodeling genes dysregulated in our models, which is an emerging phenotype of AF both in human and sheep models ${ }^{36}$.

\section{Molecular remodeling in AF with versus without AVB}

We did not expect to find a smaller number of DE genes in the AFvsCTL analysis, given our previous observation that $A F$ treatment alone without $A V B$ results in more important tissue remodeling ${ }^{10}$. One possible explanation is that our prior histological studies were done in AF animals treated for three weeks, ${ }^{10}$ whereas the results presented here reflect RNA changes after one week of AF. The transcriptomic changes in the AF+AVB group show that cells are under active chromatin modification, indicating ongoing adaptation to the stimulus. This is not observed in the AF group (lacking AVB), which may indicate that this adaptation has already occurred. This idea would be consistent with the down-regulation of chromatin-related genes recently noted in the atria of sheep $\mathrm{AF}$ models ${ }^{36}$, and may be a result of earlier establishment of profibrotic transdifferentiation in AF compared to AF+AVB canine models. 
Our analyses highlighted many genes not previously implicated in AF. While the functions of some of these genes remain uncharacterized (e.g. LENG8 in AF+AVB), we can speculate on the activities of others. For instance, TCERG1 and FNBP4, which are up-regulated in the atria of $A F+A V B$ dogs, encode co-regulated proteins that are involved, respectively, in RNA splicing and translation ${ }^{37}$. The up-regulation of these genes, with general actions on gene transcripts, may (partly) explain why more genes are dysregulated in AF+AVB animals when compared to the CTL or AF groups (Fig. 3B). Another interesting candidate is $L C O R$, which is up-regulated in the AF+AVB model and encodes a transcriptional cofactor that interacts with PPARy and RXRa to control gene expression ${ }^{38}$. While further experiments are needed to determine the extent by which $L C O R$ modifies gene expression in AF+AVB and contributes to the pathology, our RNA-seq experiments detected the dysregulation of two of its likely targets based on the literature: $C P T 1 A$ (see above) and the cell cycle regulator $C D K N 1 A^{39}$.

\section{Potential role of non-coding genes at the $D L K 1-D I O 3$ locus in early AF}

The highly-conserved DLK1-DIO3 locus hosts two differentially DNA-methylated regions modulating the expression of its non-coding RNA clusters, where in humans the maternal allele is hypomethylated with concomitant expression on the hypermethylated paternal allele of non-coding RNA and other protein-coding genes $(D L K 1, R L T 1 \text {, and } D I O 3)^{35}$. In both $A F+A V B v s C T L$ and AFvsCTL, we found a large proportion (58\% and $23 \%$, respectively) of DE miRNA at this locus, underlying its importance in AF-related adaptation. We also found dysregulation of the MEG3 IncRNA canine orthologue at this locus. MEG3 is a highly expressed IncRNA that has been studied in various pathologies, including cancer ${ }^{40}$ and more recently cardiovascular diseases ${ }^{41}$. Non-coding RNAs at this locus have been shown to mediate various cardiac developmental programs ${ }^{35}$. More specifically, MEG3 can contribute 
to the recruitment of the Polycomb repressive complex-2 (PRC2) ${ }^{42}$, a key chromatin modulating factor. Of particular interest, Mondal et al. showed that through interaction with the H3-Lys-27 methyltransferase EZH2, MEG3 can repress TGF-beta target genes, which are known to promote a profibrotic response ${ }^{43}$. Data have been presented that suggest an important role of $E Z H 2$ and/or EZH2-regulated genes in $\mathrm{AF}^{44}$.

\section{Glutamate receptor regulation by miRNAs from the $D L K 1-D I O 3$ locus}

Our GSEA analysis-predicted gene targets of DE miRNA at the DLK1-DIO3 locus suggest a role for glutamate signaling in AF. Immunostaining has confirmed the presence of glutamate receptors on cardiomyocytes ${ }^{45}$. Glutamate was also found to be significantly increased in AF patient left atrial appendages ${ }^{46}$. Glutamate signaling is important in vagal afferent neurons ${ }^{47}$, and remodeling of the glutamate system in AF may relate to the extensive previous evidence of autonomic dysfunction in AF patients ${ }^{48}$. Moreover, a recent study has shown fundamental roles for glutamatergic receptors in rat atrial cardiomyocytes and induced pluripotent stem cell-derived atrial cardiomyocytes, including a reduction in cardiomyocyte excitability after GRIA3 knockdown ${ }^{49}$. Therefore, the DLK1-DIO3 miRNA cluster may be an adaptative regulator of cardiomyocyte excitability or of neural cells in the presence of AF.

\section{Limitations}

We used cardiomyocyte-enriched samples in an attempt to obtain clearer results from the transcriptomic analysis by excluding extrinsic variability due to changes in cell composition. However, while our samples are enriched in cardiomyocytes, they do not constitute a pure cardiomyocyte preparation. A disadvantage is that variability due to changes in cell composition is not eliminated. On the other hand, our cardiomyocyte-enriched (but not pure) 
samples allow us to detect potential features of AF related to non-cardiomyocyte cells, such as autonomic dysregulation mediated by neural cells; however, we cannot unambiguously attribute DE genes to transcriptomic changes in a specific cell type. In part, we were able to control for fibroblast composition by adjustment through analysis for expression of fibroblastrelated RNA-expression patterns. Nevertheless, features underlined here should be confirmed in pure cell cultures or single cell transcriptomic assays. A second limitation is the difficulty in extrapolating our findings to gene expression changes in humans. We found only modest overlap of DE genes in our model compared to reported DE gene patterns in human; several factors could explain this (e.g. differences in biospecimen preparation, tissue heterogeneity, fundamental differences between dog and human AF pathology). It is also possible that different transcriptomic programs may be involved at the initiation of arrhythmia and tissue remodeling ( $A F$ and $A F+A V B$ dog models) when compared with those dysregulated in the atria once the pathology has been present for years.

We compared our transcriptomic results with proteomic data on the same samples and found a very high level of correlation (Fig. 2). These results are consistent with a large measure of transcriptomic control over protein expression and validate the relevance of transcriptomic analysis of these data. A further in-depth look at the proteomic signature in these models would be of interest but is beyond the scope of the present manuscript.

\section{Conclusions}

Understanding the pathophysiology of chronic human diseases such as AF is challenging because they develop over many years and initially present with only unremarkable preclinical symptoms. In this study, we took advantage of two well-characterized canine AF models to chart the transcriptomic changes that occur at the earlier phases of arrhythmia. 
bioRxiv preprint doi: https://doi.org/10.1101/2021.02.04.429512; this version posted October 19,2021. The copyright holder for this preprint

(which was not certified by peer review) is the author/funder, who has granted bioRxiv a license to display the preprint in perpetuity. It is made available under aCC-BY-NC-ND 4.0 International license.

Despite the inherent limitations in relating dog models to human AF, our results offer interesting new hypotheses for future testing, including in man. In particular, the up-regulation of miRNAs at the DLK1-DIO3 locus after 1 week of AF suggests that they may be early biomarkers of tissue remodeling and/or adaptation in the atria. 


\section{Sources of Funding}

This work was funded by the Fonds de Recherche en Santé du Québec (FRQS), the Canada Research Chair Program, the Montreal Heart Institute Foundation (MHIF), Heart and Stroke Foundation of Canada (grant \#18-0022032), Canadian Institutes of Health Research (CIHR) (grant \# 148401), the Austrian Science Fund (FWF) (projects KLI645, W1226 and F73 to RBG). F.L. received scholarships from the CIHR, FRQS, MHIF and Université de Montréal.

\section{Disclosures}

None

\section{Supplemental Materials}

Supplementary Table I \& Supplementary Figures I-II

Supplementary Tables II-VI 


\section{References}

1. Andrade J, Khairy P, Dobrev D and Nattel S. The clinical profile and pathophysiology of atrial fibrillation: relationships among clinical features, epidemiology, and mechanisms. Circ Res. 2014;114:1453-68.

2. Heijman J, Algalarrondo V, Voigt N, Melka J, Wehrens XH, Dobrev D and Nattel S. The value of basic research insights into atrial fibrillation mechanisms as a guide to therapeutic innovation: a critical analysis. Cardiovasc Res. 2016;109:467-79.

3. Wijffels MC, Kirchhof CJ, Dorland R and Allessie MA. Atrial fibrillation begets atrial fibrillation: a study in awake chronically instrumented goats. Circulation. 1995;92:1954-1968.

4. Wakili R, Voigt N, Kaab S, Dobrev D and Nattel S. Recent advances in the molecular pathophysiology of atrial fibrillation. J Clin Invest. 2011;121:2955-68.

5. Nattel S and Harada M. Atrial remodeling and atrial fibrillation: recent advances and translational perspectives. J Am Coll Cardiol. 2014;63:2335-45.

6. Ma N, Lu R, Zhao D, Jiang Z, Tang M, Bao C and Mei J. Left Atrial Appendage Fibrosis and 3Year Clinical Outcomes in Atrial Fibrillation After Endoscopic Ablation: A Histologic Analysis. Ann Thorac Surg. 2020;109:69-76.

7. Burstein B, Qi XY, Yeh YH, Calderone A and Nattel S. Atrial cardiomyocyte tachycardia alters cardiac fibroblast function: a novel consideration in atrial remodeling. Cardiovasc Res. 2007;76:442-52.

8. Raymond-Paquin A, Nattel S, Wakili R and Tadros R. Mechanisms and Clinical Significance of Arrhythmia-Induced Cardiomyopathy. Can J Cardiol. 2018;34:1449-1460.

9. DiMarco JP. Atrial fibrillation and acute decompensated heart failure. Circ Heart Fail. 2009;2:72-3.

10. Guichard JB, Xiong F, Qi XY, et al. Role of atrial arrhythmia and ventricular response in atrial fibrillation induced atrial remodeling. Cardiovasc Res. 2020.

11. Wood MA, Brown-Mahoney C, Kay GN and Ellenbogen KA. Clinical outcomes after ablation and pacing therapy for atrial fibrillation: a meta-analysis. Circulation. 2000;101:1138-1144.

12. Harada M, Luo X, Qi XY, et al. Transient receptor potential canonical-3 channel-dependent fibroblast regulation in atrial fibrillation. Circulation. 2012;126:2051-64.

13. Zhang D, Wu C-T, Qi X, et al. Activation of histone deacetylase- 6 induces contractile dysfunction through derailment of $\alpha$-tubulin proteostasis in experimental and human atrial fibrillation. Circulation. 2014;129:346-358.

14. Brundel BJ, Shiroshita-Takeshita A, Qi X, Yeh Y-H, Chartier D, van Gelder IC, Henning RH, Kampinga $\mathrm{HH}$ and Nattel S. Induction of heat shock response protects the heart against atrial fibrillation. Circulation research. 2006;99:1394-1402. 
15. Qi X-Y, Diness JG, Brundel BJ, et al. Role of small-conductance calcium-activated potassium channels in atrial electrophysiology and fibrillation in the dog. Circulation. 2014;129:430-440.

16. Bray NL, Pimentel H, Melsted P and Pachter L. Near-optimal probabilistic RNA-seq quantification. Nature biotechnology. 2016;34:525-527.

17. Soneson C, Love MI and Robinson MD. Differential analyses for RNA-seq: transcript-level estimates improve gene-level inferences. F1000Research. 2015;4.

18. Love MI, Huber W and Anders S. Moderated estimation of fold change and dispersion for RNA-seq data with DESeq2. Genome Biol. 2014;15:550.

19. Ritchie ME, Phipson B, Wu D, Hu Y, Law CW, Shi W and Smyth GK. limma powers differential expression analyses for RNA-sequencing and microarray studies. Nucleic acids research. 2015;43:e47-e47.

20. Chen S, Zhou Y, Chen Y and Gu J. fastp: an ultra-fast all-in-one FASTQ preprocessor. Bioinformatics. 2018;34:i884-i890.

21. Dobin A, Davis CA, Schlesinger F, Drenkow J, Zaleski C, Jha S, Batut P, Chaisson M and Gingeras TR. STAR: ultrafast universal RNA-seq aligner. Bioinformatics. 2013;29:15-21.

22. ENCODE. ENCODE_miRNA-seq_STAR_parameters 2020.

23. Donovan MKR, D'Antonio-Chronowska A, D'Antonio M and Frazer KA. Cellular deconvolution of GTEx tissues powers discovery of disease and cell-type associated regulatory variants. Nat Commun. 2020;11:955.

24. Newman AM, Steen CB, Liu CL, et al. Determining cell type abundance and expression from bulk tissues with digital cytometry. Nat Biotechnol. 2019;37:773-782.

25. Shen N-N, Zhang Z-L, Li Z, Zhang C, Li H, Wang J-L, Wang J and Gu Z-C. Identification of microRNA biomarkers in atrial fibrillation: A protocol for systematic review and bioinformatics analysis. Medicine. 2019;98.

26. Hsu J, Gore-Panter S, Tchou G, et al. Genetic control of left atrial gene expression yields insights into the genetic susceptibility for atrial fibrillation. Circulation: Genomic and Precision Medicine. 2018;11:e002107.

27. Rath S, Sharma R, Gupta R, et al. MitoCarta3. 0: an updated mitochondrial proteome now with sub-organelle localization and pathway annotations. Nucleic Acids Research. 2021;49:D1541-D1547.

28. Cox J, Hein MY, Luber CA, Paron I, Nagaraj N and Mann M. Accurate proteome-wide labelfree quantification by delayed normalization and maximal peptide ratio extraction, termed MaxLFQ. Mol Cell Proteomics. 2014;13:2513-26.

29. Chen C-L, Lin J-L, Lai L-P, Pan C-H, Huang SKS and Lin C-S. Altered expression of FHL1, CARP, TSC-22 and P311 provide insights into complex transcriptional regulation in pacing- 
induced atrial fibrillation. Biochimica et Biophysica Acta (BBA)-Molecular Basis of Disease. 2007;1772:317-329.

30. Ding Y, Yang J, Chen P, et al. Knockout of SORBS2 Protein Disrupts the Structural Integrity of Intercalated Disc and Manifests Features of Arrhythmogenic Cardiomyopathy. J Am Heart Assoc. 2020;9:e017055.

31. Olson TM, Alekseev AE, Liu XK, et al. Kv1. 5 channelopathy due to KCNA5 loss-of-function mutation causes human atrial fibrillation. Human molecular genetics. 2006;15:2185-2191.

32. Lin H, Yin X, Xie Z, et al. Methylome-wide association study of atrial fibrillation in Framingham Heart Study. Scientific reports. 2017;7:40377.

33. Hall AW, Chaffin M, Roselli C, et al. Epigenetic Analyses of Human Left Atrial Tissue Identifies Gene Networks Underlying Atrial Fibrillation. Circulation: Genomic and Precision Medicine. 2020;13:e003085.

34. Luo X, Yang B and Nattel S. MicroRNAs and atrial fibrillation: mechanisms and translational potential. Nature Reviews Cardiology. 2015;12:80.

35. Dill TL and Naya FJ. A hearty dose of noncoding RNAs: The imprinted DLK1-DI03 locus in cardiac development and disease. Journal of cardiovascular development and disease. 2018;5:37.

36. Alvarez-Franco A, Rouco R, Ramirez RJ, et al. Transcriptome and proteome mapping in the sheep atria reveal molecular features of atrial fibrillation progression. Cardiovascular Research. 2020.

37. Deelen P, van Dam S, Herkert JC, et al. Improving the diagnostic yield of exome-sequencing by predicting gene-phenotype associations using large-scale gene expression analysis. Nature communications. 2019;10:1-13.

38. Shalom-Barak T, Liersemann J, Memari B, et al. Ligand-dependent corepressor (LCoR) is a rexinoid-inhibited peroxisome proliferator-activated receptor $\gamma$-retinoid X receptor $\alpha$ coactivator. Molecular and cellular biology. 2018;38.

39. Calderon MR, Verway M, An B-S, DiFeo A, Bismar TA, Ann DK, Martignetti JA, ShalomBarak T and White JH. Ligand-dependent Corepressor (LCoR) Recruitment by Krüppel-like Factor 6 (KLF6) regulates expression of the cyclin-dependent kinase inhibitor CDKN1A gene. Journal of Biological Chemistry. 2012;287:8662-8674.

40. Zhou Y, Zhang X and Klibanski A. MEG3 noncoding RNA: a tumor suppressor. Journal of molecular endocrinology. 2012;48:R45-R53.

41. Kumar S, Williams D, Sur S, Wang J-Y and Jo H. Role of flow-sensitive microRNAs and long noncoding RNAs in vascular dysfunction and atherosclerosis. Vascular pharmacology. 2019;114:76-92. 
42. Kaneko S, Bonasio R, Saldaña-Meyer R, Yoshida T, Son J, Nishino K, Umezawa A and Reinberg D. Interactions between JARID2 and noncoding RNAs regulate PRC2 recruitment to chromatin. Molecular cell. 2014;53:290-300.

43. Mondal T, Subhash S, Vaid R, et al. MEG3 long noncoding RNA regulates the TGF- $\beta$ pathway genes through formation of RNA-DNA triplex structures. Nature communications. 2015;6:7743.

44. Song S, Zhang R, Mo B, et al. EZH2 as a novel therapeutic target for atrial fibrosis and atrial fibrillation. Journal of molecular and cellular cardiology. 2019;135:119-133.

45. Gill S, Veinot J, Kavanagh M and Pulido 0. Human heart glutamate receptors-implications for toxicology, food safety, and drug discovery. Toxicologic pathology. 2007;35:411-417.

46. Lai S, Hua X, Gao R, Zeng L, Song J, Liu J and Zhang J. Combinational Biomarkers for Atrial Fibrillation Derived from Atrial Appendage and Plasma Metabolomics Analysis. Scientific reports. 2018;8:1-11.

47. de Lartigue G. Putative roles of neuropeptides in vagal afferent signaling. Physiology \& behavior. 2014;136:155-169.

48. Agarwal SK, Norby FL, Whitsel EA, et al. Cardiac autonomic dysfunction and incidence of atrial fibrillation in a large population-based cohort. Journal of the American College of Cardiology. 2017;69:291.

49. Xie D, Xiong K, Su X, et al. Identification of an endogenous glutamatergic transmitter system controlling excitability and conductivity of atrial cardiomyocytes. Cell Research. 2021:114. 
Figure 1. Deconvolution of canine atria cell composition using bulk RNA-sequencing.

(A) We inferred cell fractions with CIBERSORTx and an atrial-specific gene signature matrix obtained using orthologous murine genes ${ }^{23}$. We present cell fractions for each dog sample that we analyzed in this study. CTL, control; AF, Atrial-tachypacing; AF+AVB, AF with AtrioVentricular Block. (B) When we group animals per treatment arm, we observed a significantly higher fraction of fibroblasts in the atrial fibrillation dog models (AF and AF+AVB) than in the control animals (AFvsCTL Wilcoxon's test $P=0.0087$ and AF+AVBvsCTL $P=0.015$ ). Principal component analysis of the top 1000 most variable genes expressed in canine atria before (C) and after (D) correction for fibroblast fraction show treatment-dependent clustering after correction for cell composition. 
Figure 2. Validation of highly expressed RNA by proteomics. (A) In 18 atrial samples, 755 genes (N.S.=619, RNA.sign=122, Both.sign=14) found in both datasets are highly correlated at the protein (x-axis) and RNA (y-axis) levels (Pearson's $r=0.49, P=1.57 \times 10^{-46}$ ). For reference, we annotated 15 genes that are differentially expressed in the RNA-seq experiment and have high protein expression levels. N.S., not differentially expressed in the RNA-seq or proteomic experiment; RNA.sign, genes that are differentially expressed in the RNA-seq assay only; Both.sign, differentially expressed genes in both the RNA-seq and proteomic experiments. DE genes in the RNAseq dataset have an FDR $<0.01$ (likelihood ratio test) and proteomics dataset an FDR $<0.05$ (F-test). The grey area around the line corresponds to the $95 \%$ confidence interval. (B) Relative expression level of all transcripts measured in the RNAseq experiment. The histogram shows that genes that are present in both the RNA-seq and proteomic experiment are highly expressed (Common, dark grey) in comparison to the expression levels of all transcripts measured (all_RNA, light grey). 
Figure 3. Analyses of differentially expressed atrial genes identify many biological pathways that are dysregulated in atrial fibrillation dog models. (A) Volcano plots of all transcripts that we analyzed in this study. Transcripts in red have a false discovery rate $(F D R)<0.01$. We found 434,5971 and 7867 genes that were DE in the AFvsCTL, $A F+A V B v s C T L$, and $A F v s A F+A V B$ analyses, respectively. The full DE results are available in Supplemental Table II. (B) Upset plot showing the intersection of up -and down-regulated DE genes $(F D R<0.01)$ in each analysis. (C) The five most significant biological pathways identified using gene-set enrichment analyses (GSEA) for each set of DE genes (FDR $<0.01)$. Full results are available in Supplemental Table IV. 
Figure 4. 11 differentially expressed microRNAs (miRNAs) map to a canine chromosome 8 region that is syntenic to human DLK1-DIO3. (A) Volcano plots of all miRNA that we measured in our experiments. We identified 31, 19 and 20 miRNA that are differentially expressed (false discovery rate $(F D R)<0.01$ ) in the AFvsCTL, AF+AVBvsCTL and AFvsAF+AVB analyses, respectively. (B) Miami plots of miRNA and their corresponding statistical significance (y-axis) for the AF+AVBvsCTL (top) and ATvsCTL (bottom) analyses. An arrow indicates the miRNA cluster located on the canine chromosome 8 region that is syntenic to human DLK1-DIO3. The odd and even chromosomes FDR values are in blue and red respectively. (C) Upset plot showing the DE miRNA targets located in the syntenic DLK1-DIO3locus and their corresponding number of potential target RNA. We identified potential targets with the MiRNAtap package (predicted by $\geq 3$ databases) from DE miRNA (FDR <0.01) and DE mRNA (FDR<0.01). (D) Gene-set enrichment analyses (GSEA) with the potential gene targets (x-axis) of the DE miRNA located at the syntenic DLK1-DIO3 locus. We only present the top five pathways enriched in this analysis. A red square in the heatmap indicates membership of a given target gene to the biological pathways located on the left (empty columns were removed for clarity). GSEA FDR and AF+AVBvsCTL DE FDR are on the right and top of the heatmap, respectively. 
bioRxiv preprint doi: https://doi.org/10.1101/2021.02.04.429512; this version posted October 19,2021 . The copyright holder for this preprint

(which was not certified by peer review) is the author/funder, who has granted bioRxiv a license to display the preprint in perpetuity. It is made available under aCC-BY-NC-ND 4.0 International license.

Figure 5. Overlaps in genes differentially expressed in canine AF models and human AF patients. We compared differentially expressed genes in our canine AF models with annotated human orthologues that are DE in human AF left atrial appendages ${ }^{26}$. Homo Sapiens; hsa, hsa_AF; AF in AF rhythm, hsa_AF.SR; AF in sinus rhythm, hsa_CTL; no AF. 


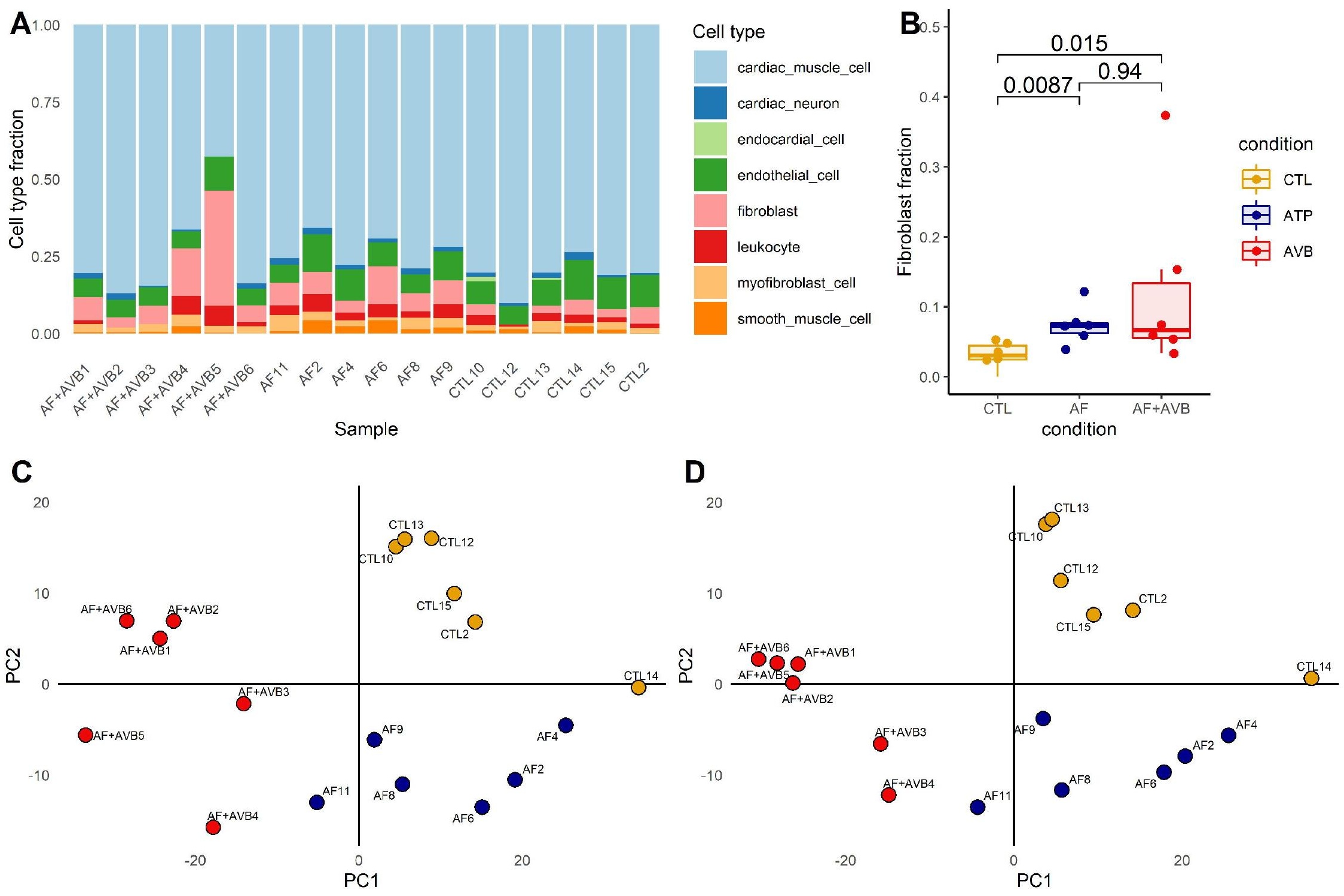




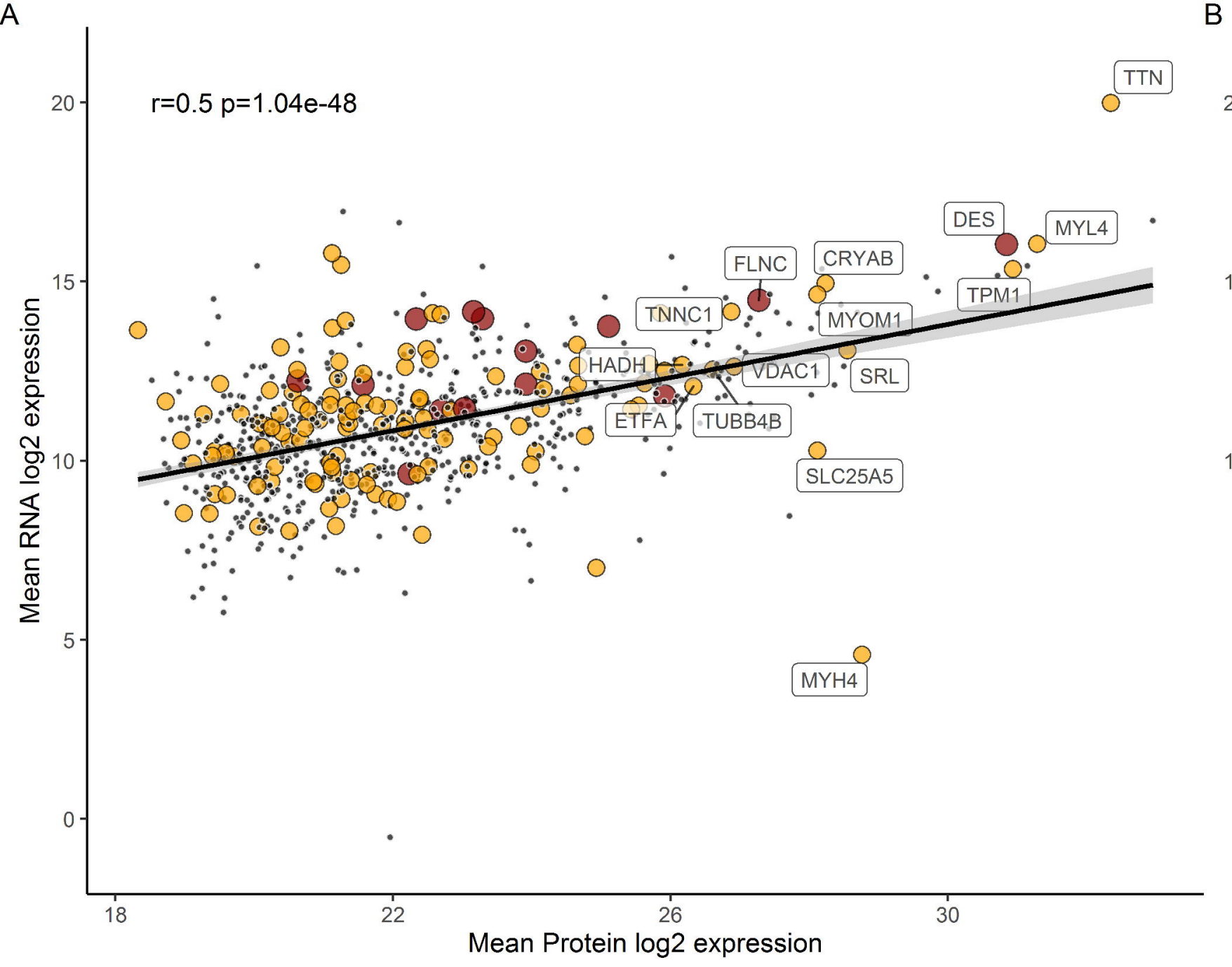

B

0

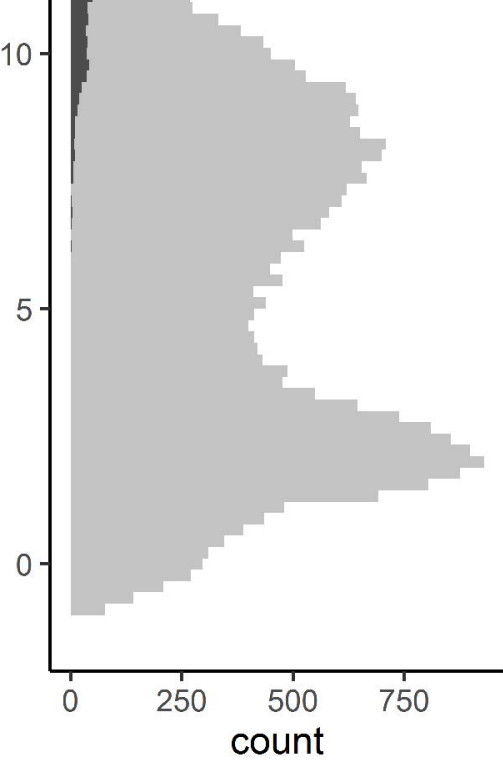

- N.S. $\bigcirc$ RNA.sign $\bigcirc$ Both.sign

all_RNA

Common 
$20=$

$10=$

$0=$ $-5.0$

\section{$\uparrow \mathrm{CTL}$}

$20=$

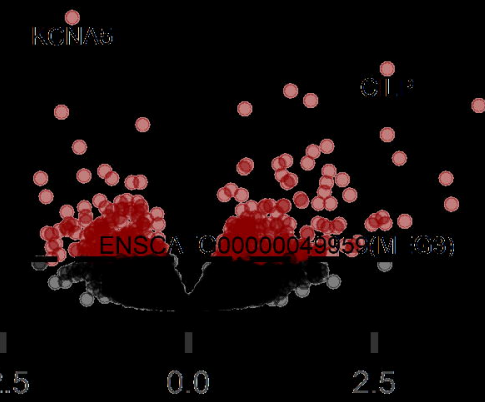

Cof fold the the

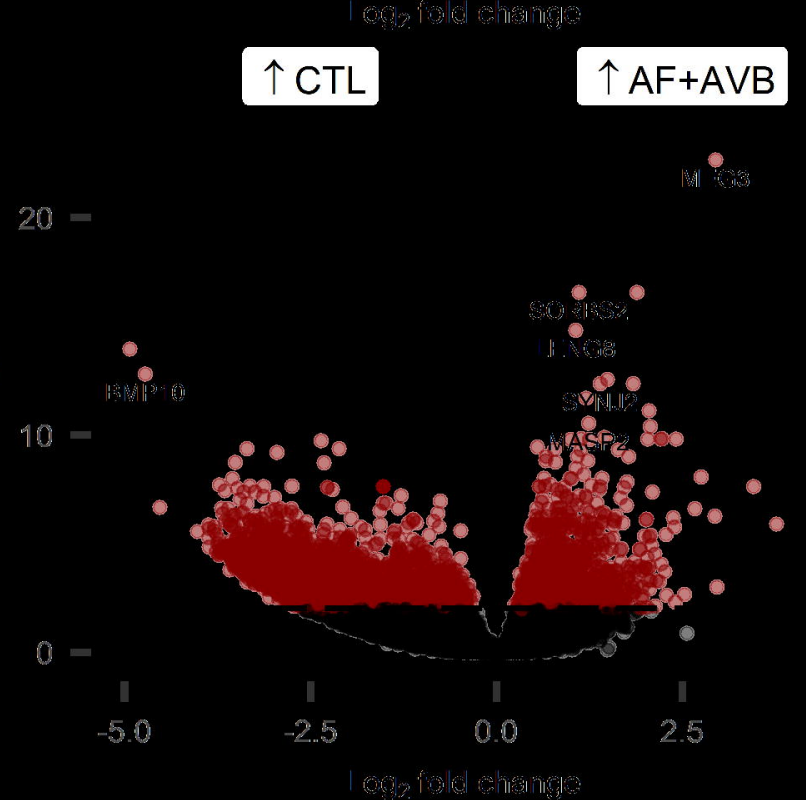

$\uparrow A F \quad \uparrow A F+A V B$

$20=$
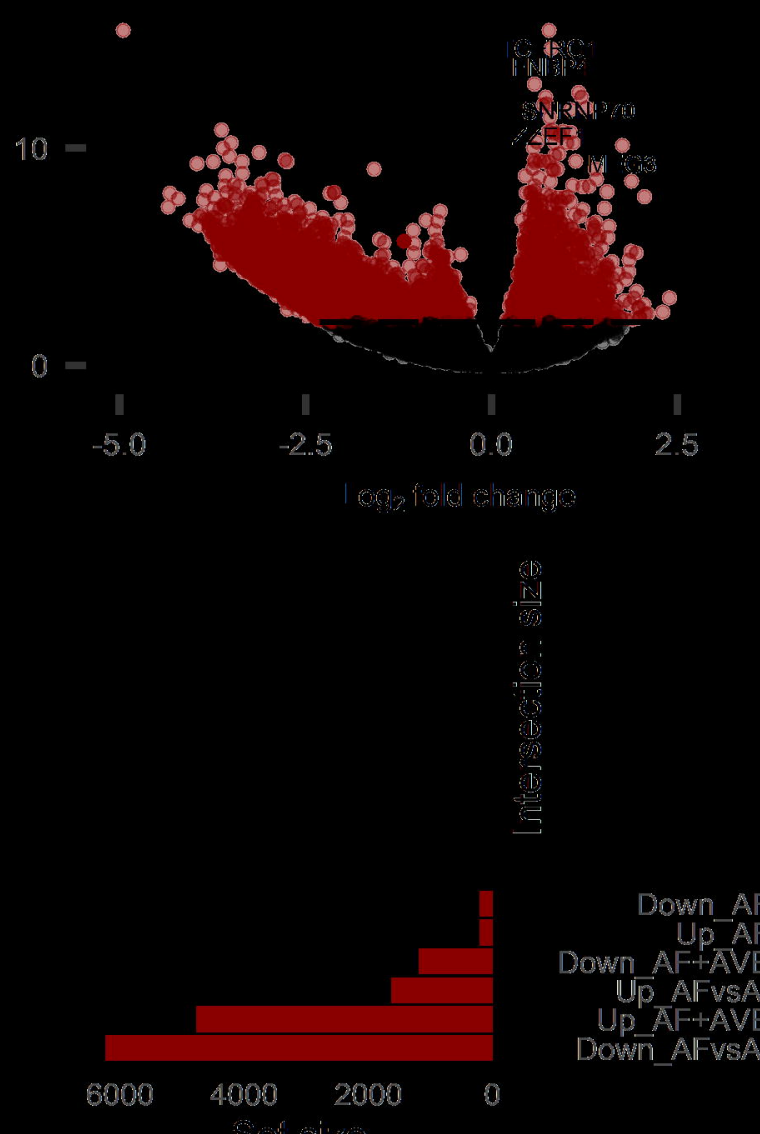

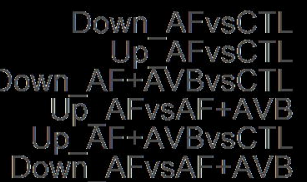

$$
\begin{array}{r}
\text { circulator_system_development } \\
\text { raswoss. to wounding } \\
\text { tube_development }
\end{array}
$$

circulatory system dovelogment

cardiovascular system develonment

regulation_of_cellular_component_movement

blood_vessel_morphogenesis

regulation_of_anatomical_structure_marphogenesis

extracellular Structure onganization

musche structure development

chemical_homeostasis

homeostatic_process

striated_muscle_cell_differentiation

nervous system process

sensery_perception

ion_transport

cotranstational_protein_largeting_to_membrane

9_protein_coupled_receptor_signaling_pathway

nervous_system_process

sensory_perception

9_proteir_coupled_receptor_signaling_pathway

ion transpor

sensory_perception_of_chemicall_stimulus covalent_chromatin_modification

chromalin grganization

positive_regulation_of_nucleobase_containing_compound_metabolic_process

positive_regulation_of_ma_metabolic_process

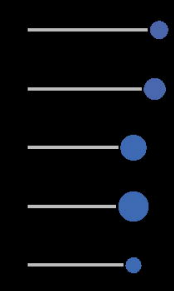

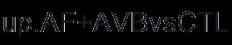

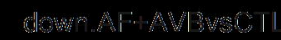

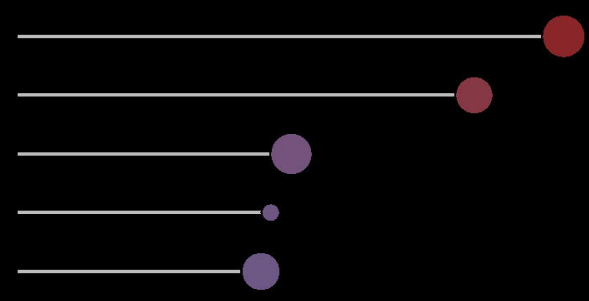

WOA FWA AFHA

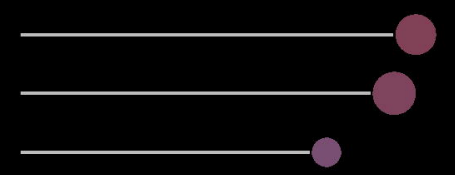

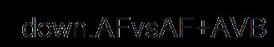

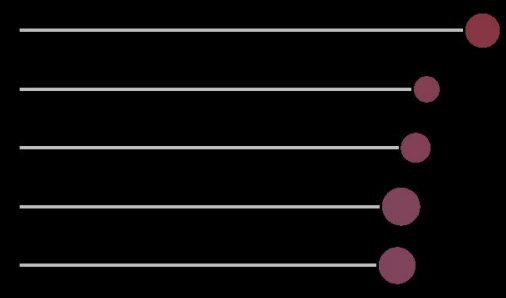

30

40

19019412
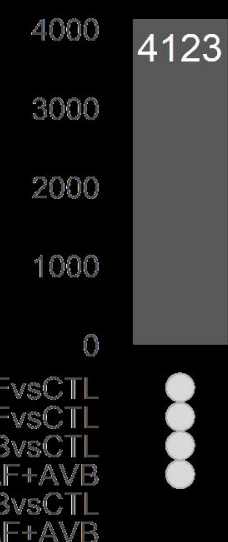


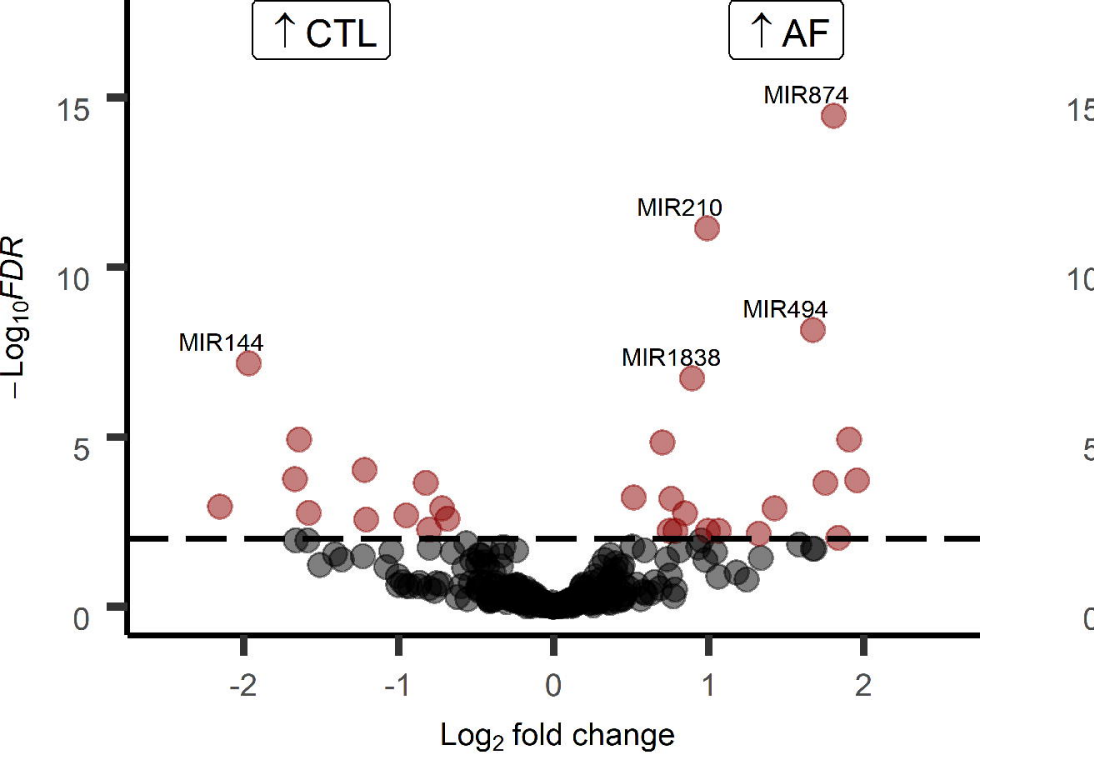

$A F+A V B v s C T L$

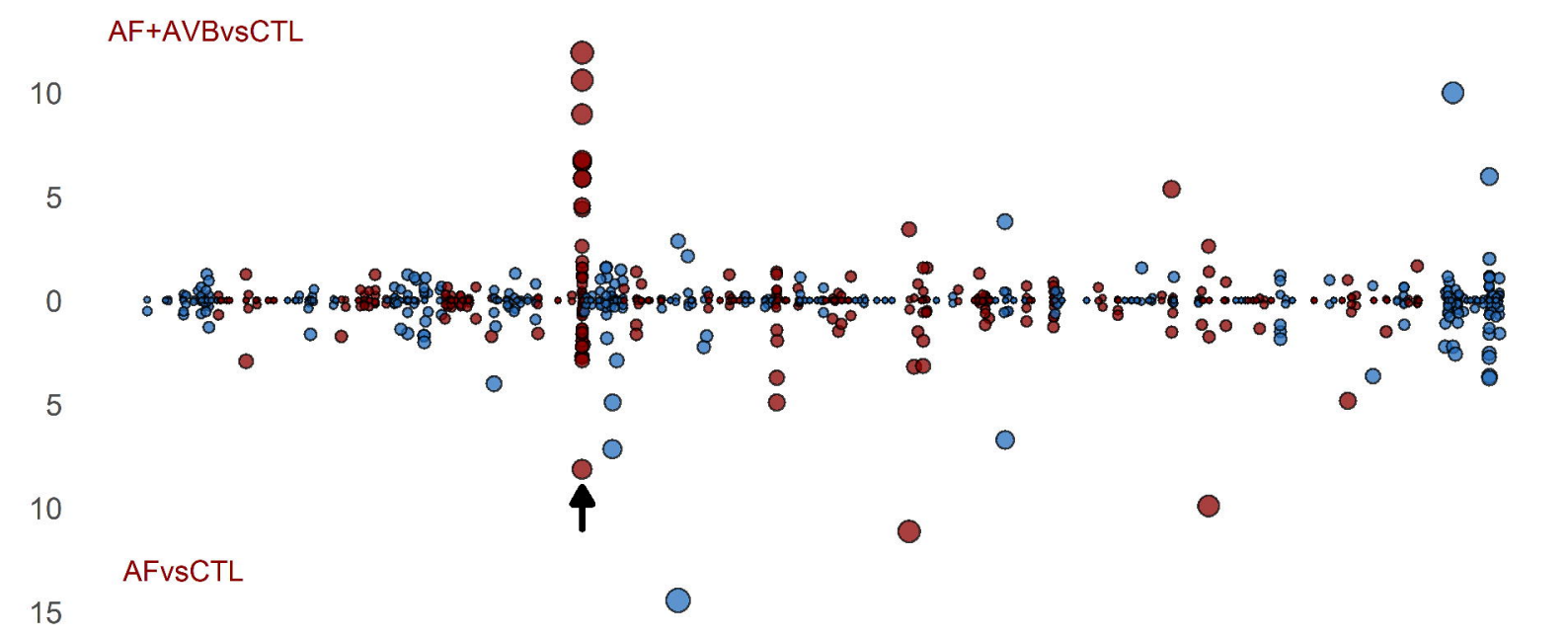

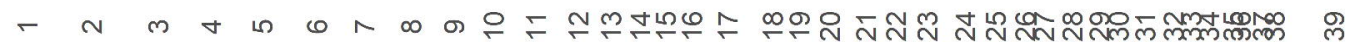

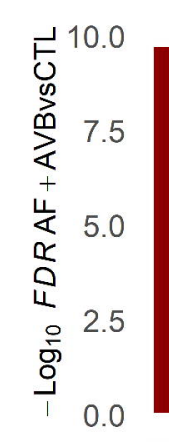

GO_Synaptic transmission glutamatergic GO_Cell_cell_signaling GO_Behavior GO Synaptic signaling GO_Glutamate_receptor_signaling_pathway

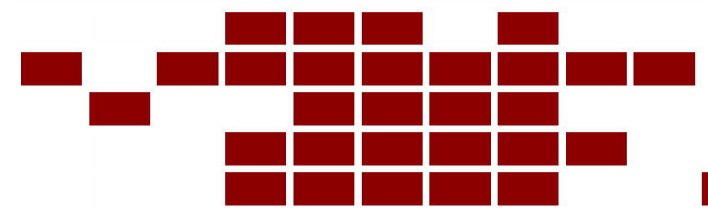

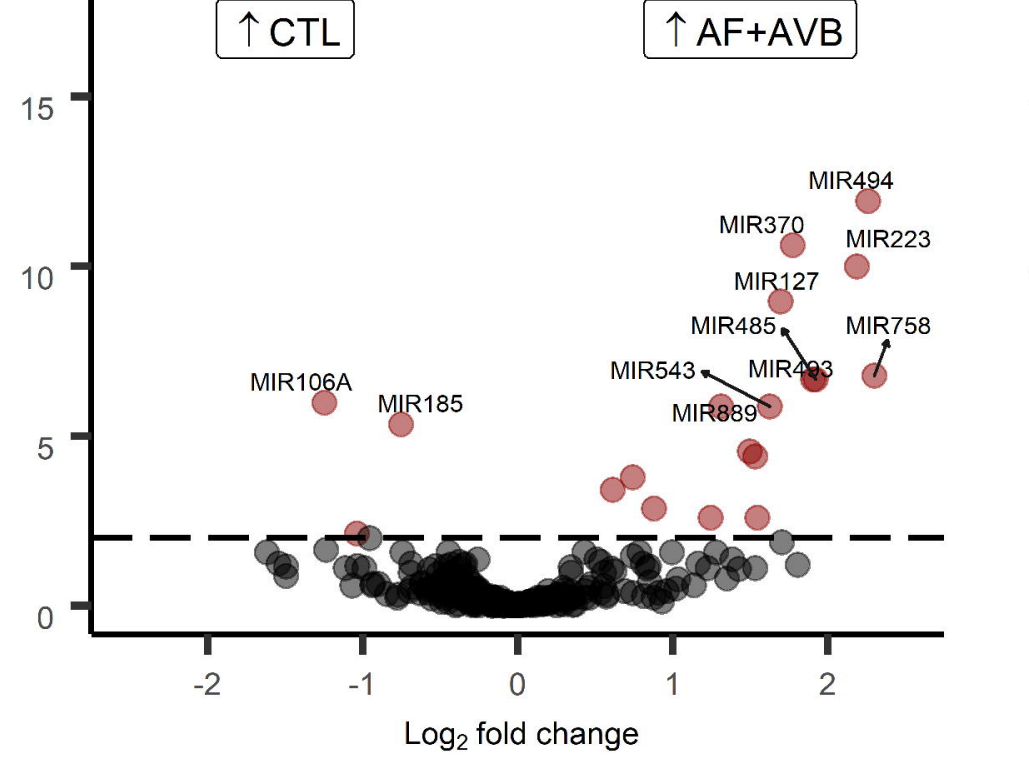

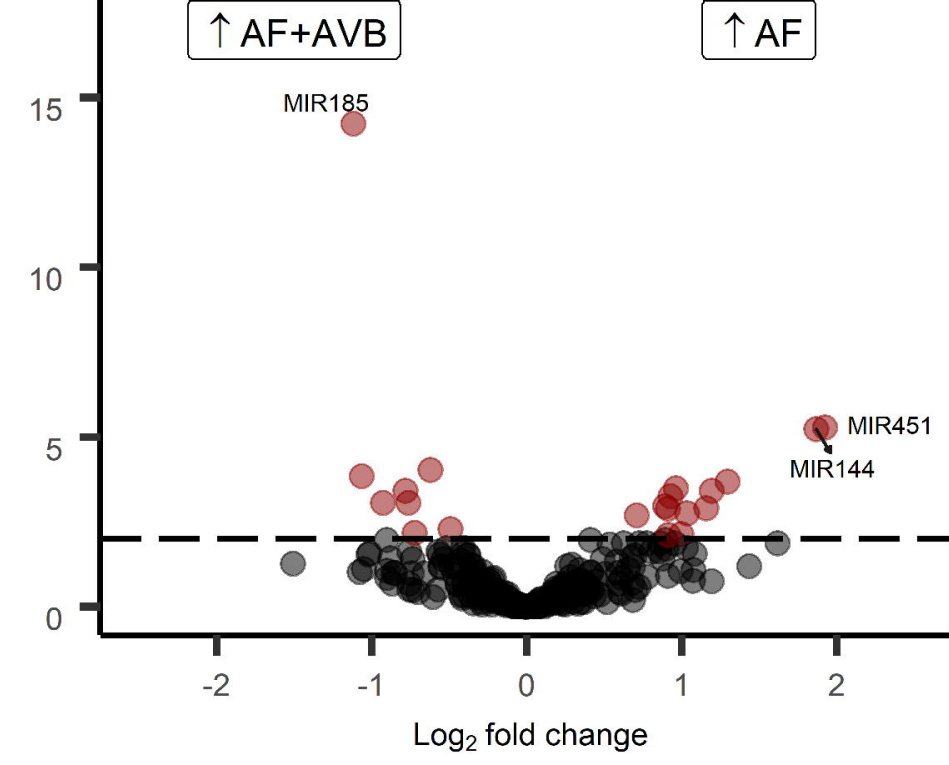

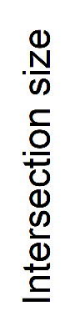

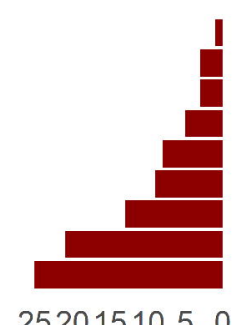

252015105

Set size
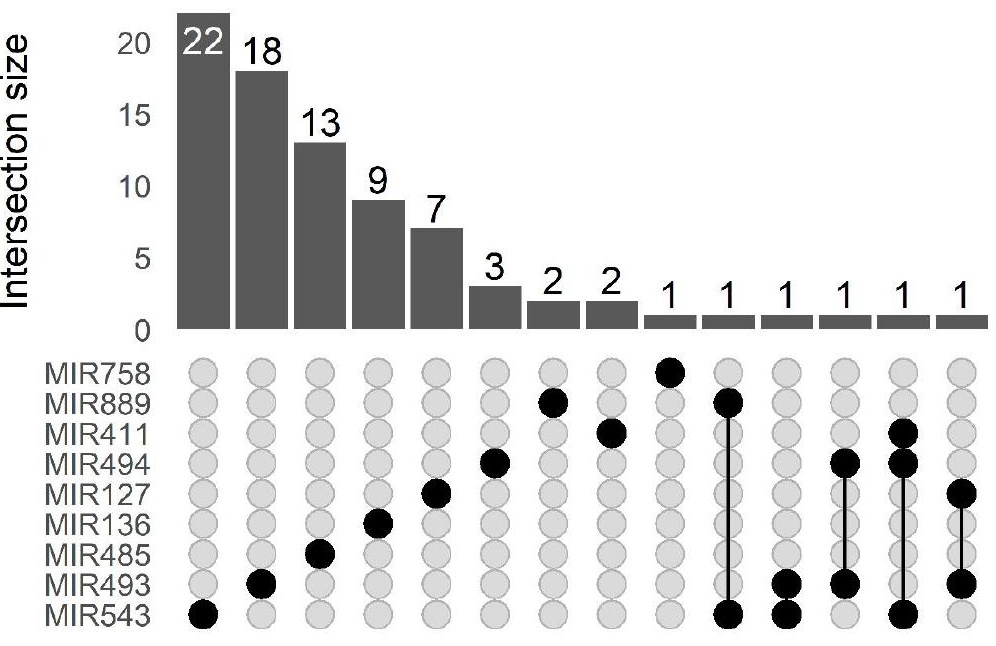

group
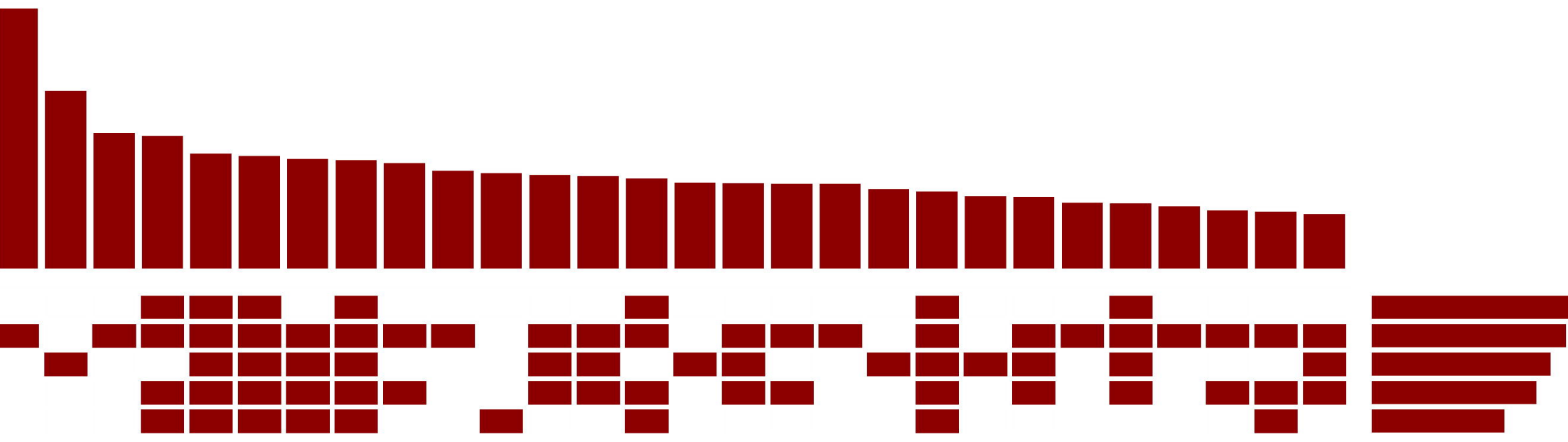

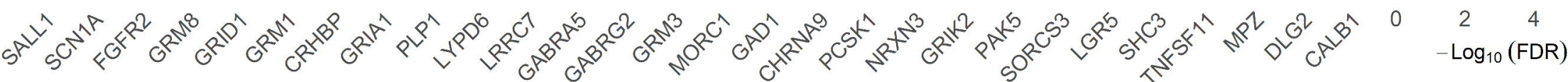



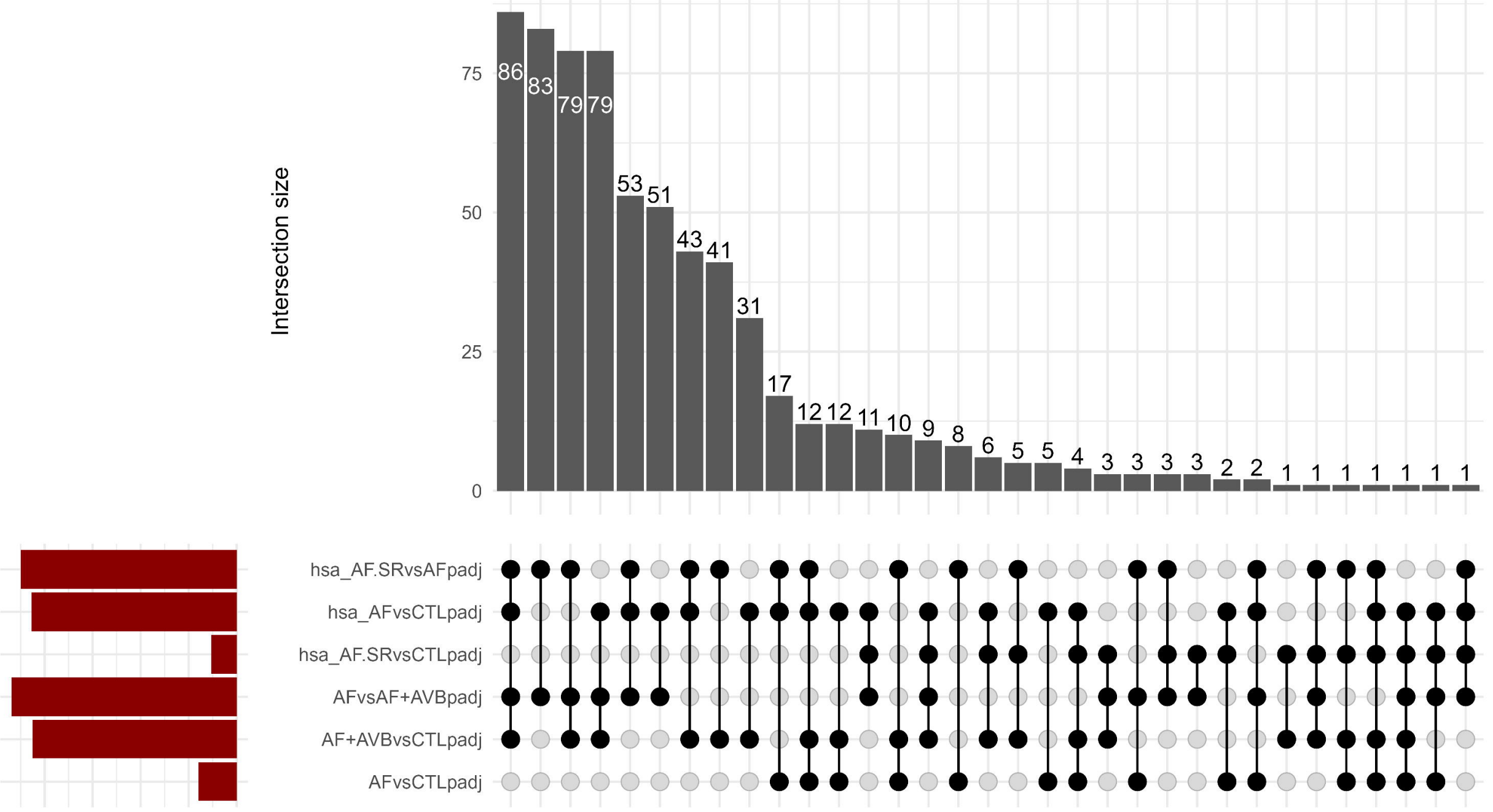

$\begin{array}{lllll}400 & 300 & 200 & 100 & 0\end{array}$ 


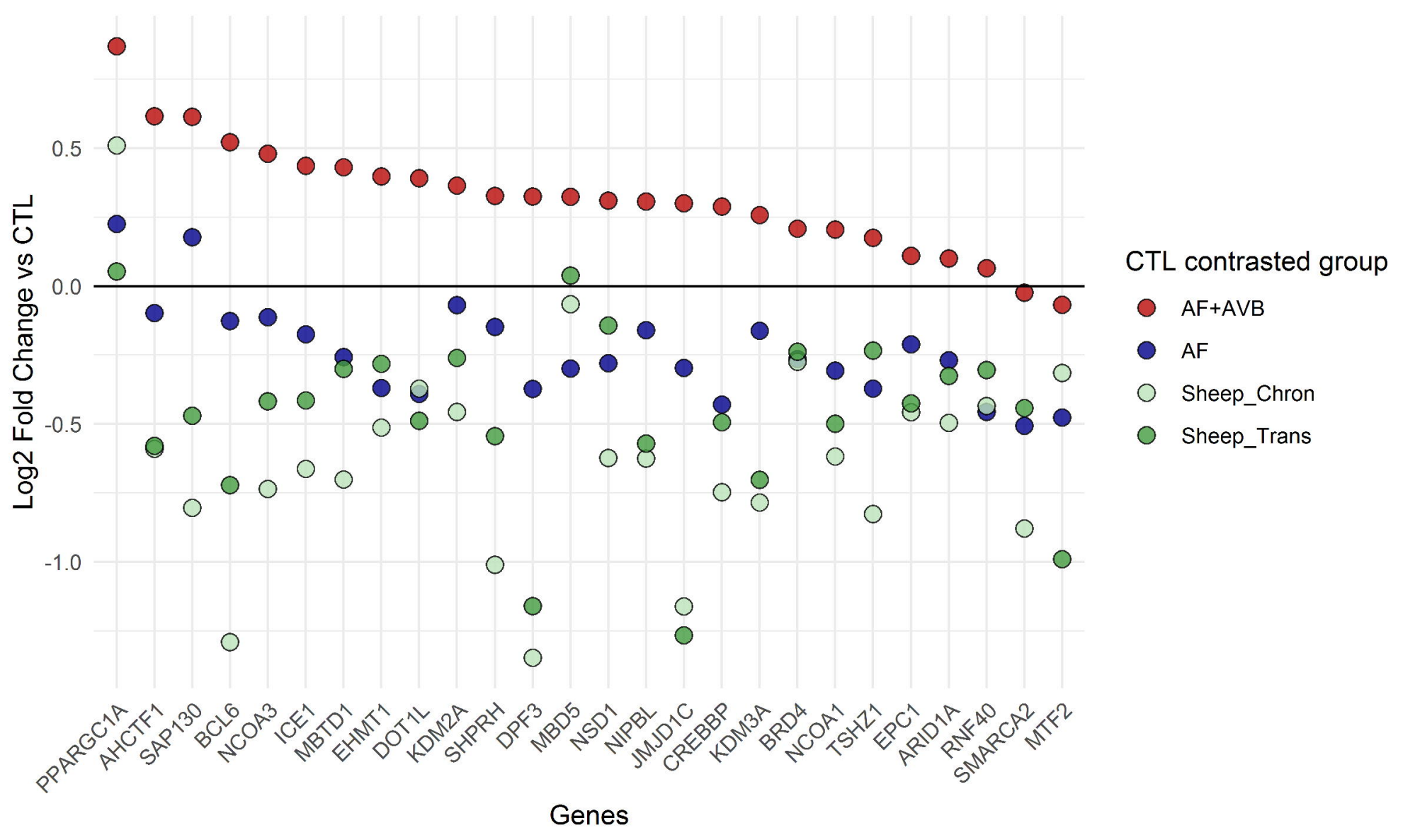

